\title{
Sachregister zu Band 81
}

Die fett gedruckten Zahlen bedeuten Eigenarbeiten. Bb. = Buchbesprechungen.

A

Ablatio retinae und Unfall 95

Heilungsvorgänge bei der ex-perimentellen - 104

Heilungsversuche bei progno-stisch ungünstigen Fallen von - 277

Behandlung der -357

Operation der - 357

durch Verkürzungsoperation ge-heilt in

nachträgliche Heilung eines Fal-les von - 186

Akkommodationslähmung,

angeborene 356 Albinismus solum bulbi 367 Albinotisches Auge 173 Allergische

Hautreaktion, Ein-

fluß der Ostseeheilfaktoren auf

die - 115 Aniridie, scheinbare - post par-turn mit Embryotoxon posterius

bei Hydrophthalmus congenitus

48 Anophthalmus mit Orbital-Ober-

lidzyste $5 \delta$ Argyll-Robertson, Aus- und

Rückbildungsdauer eines Falles

von - 66 Arteria hyaloidea persistens,

seltener Fall von -367

- $\quad$ eigenartige Reste der - 190Arzt als Zeuge in der Sozialver-

sicherung und Reichsversorgung

276 (Bb.) Atmungsvorgänge der Gewebe

des Auges 92 Auge, das menschliche - und seine

Erbanlagen 275 (Bb.) Augenheilkunde, Entwicklung

der-im 18. u. 19. Jahrhundert

$375(\mathrm{Bb})$. Augenkinegraph 346 Augenkrankheiten, neurologi-

sche Beziehungen der - 363 Augenlider s. Lider Augenmuskellähmungen 349 - bei

Basedowscher Krankheit 104 Augenoperationen, ambulant

ausgeführte - 89 Augenverletzungen, Hacken-

splitterverl. im Bodenseegebiet

319

- $\quad$ Denigsche Operation bei chemi-schen - 342

Avitaminose, Hornhautverände-rungen bei $-\cdot 82$

B

Basedowsche Krankheit, Au-genmuskellähmung bei - 104 Basophilismus, hypophysärer 193

Beweglichkeitsstörungen, angeborene-- der Augenmuskeln

$35 \mathrm{i}$

Bewegungsapparat des Auges 345 (Bericht) 
Bindehaut s. Conjunctiva

Blickkrämpfe 349

Blepharochalasis, operative Behandlung gegen - 366

Blicklähmung, vertikale 349

- bei Basedowscher Krankheit 104Blut-Kammerwasserschranke,

Kohlehydratuntersuchungen zur Frage der - 190 Brillantgrün bei Ulcus serpens 77

C Canalis opticus 268 Carotis interna und Sehnerv 92 Chalazion, ein cornu cutaneum-

ähnliches -106 Cholesterinablagerung in der

Vorderkammer 112 Chorioidea, spontane Ablösung

der - 224 Chorioidealkolobom mit Glia-

wucherungen unter dem Bilde

des Pseudoglioms 155 Conjunctiva, Knötchenbildung

der --und ihre Beziehung zu den

Rheumatismusknötchen 19 Conjunctivadeckung nach

Kuhnt 84

- $\quad$ technische Weiterbildung der -239

Conjunctivitis hyperplastica plasmacellularis 355

Cornea, Pathologie der - 70 (Bericht)

- multiple Abszesse der -108

Entartung der -80

Tuberkulose der -108

eigenartige Viruserkrankung bei-

der -188

- $\quad$ Variabilität der D.urchmesserund der Oberflächengröße der -bei Megalo-, Normal- und Mikro-C. 132

- - Oberflächengröße der - beim Embryo und Neugeborenen und ihre Beziehungen zur Megalocornea der Erwachsenen 213

- $\quad$ Knötchenbildung der - - undihre Beziehung zu den Rheumatismusknötchen 19

378

Sachregister

zu Band 81.

Cornea, Maßnahmen an den i Augenlidern zum Schutze der - bei Glotzaugen und Fazialis-

lähmung 13

- $\quad$ Tätowage der -· mit Gold-chlorid 356

Corneatrübung, nur familiar auf-

tretende - 359 Corneatransplantation 362 Cyanosis retinae 363

D

Dalrymplesches Zeichen 360 Denigsche Operation bei chemi-

schen Verletzungen des Auges

342 Dentale Infektion, Iritis bei

93 Druck, intraokularer, Bestim-

mung der täglichen Schwankun-

gen des -178 Dysostosis craniofacialis 268

E

Ektopia lentis, durch 4 Genera-tionen vererbt 149

Elliotsche Operation 117, 182 
Embryotoxon posterius bei Hydrophthalmus congenitus mit scheinbarer Aniridie post par-turn 48

Endarteriitis obliterans durch Kälteschädigung und nach Trauma 272

Entoptoskopie 244

Enzephalitis lethargica, Moti-litätsstörungen bei - 349

Episklera, Pathologie der -87 (Bericht)

Epitheldystrophie, Fuchssche -81

Erbanlagen des menschlichen Auges 275 (Bb.)

Exophthalmus 262

- $\quad$ Phlebolithen bei intermittieren-dem - 91

Exzision, kombinierte oder Tar-soplastik 108

$\Gamma$

Fazialislähmung,Maßnahmenan den Augenbildern zum Schutze der Hornhaut bei - 13

Feuerlamelle 258

Fieberbehandlung bei Keratitis parenchymatosa 72

Fluoreszenzmikroskop 113

Fokale Infektion 173

Fusion, auf welcher Bahn spielt sich die - ab ? 142

G

Gehirntumor mit Lähmung des Nervus VI, durch hypertonische Injektionen gebessert 374

I Glaskörper 260 (Bericht) Glaskörperablösung, ringförmi-

ge hintere - 363 Glaukom 178 (Bericht) Glaukomoperationen 182 - vergleichende Bewertung der -

117 Glotzaugen, Maßnahmen an den

Augenlidern zum Schutze der

Hornhaut bei - 13 Goldchlorid, Hornhauttätowage

mit - 356 Goldimpragnation bei Ulcusser-

pens 76 Granulomatose, Lipoid-G. 372 Grenzstrahlen bei Glaukom 181

- bei Ulcus rodens 78Großhirnhemisphäre, besonde-

rer Symptomenkomplex bei Tu-moren im Bereich einer - 274

Grubenbildung, umschriebene - des Sehnervenkopfes 314

Gumma der Orbita 112

$\mathrm{H}$

Hackensplitterverletzungen

des Auges im Bodenseegebiet

319 Haftgläser, besondere Forme n

geschliffener - 99 Hemianopsie, homonyme und

Spontangangrän an der unteren

Extremität 272 Herpes corneae 74 Herpes iridis 175 Heterochromie 172 -- Hornersyndrom und

Status dys-

trophicus 113 Heterophorien, Messung der -

347 Hornersyndrom, Heterochromie

und Status dystrophicus 113 Hornhaut s. Cornea. Hydrophthalmus, Ergebnisse

der Behandlung des - 271

- $\quad$ Embryotoxon posterius bei H.congenitus mit scheinbarer Aniridie post partum 48

Hyperthermie 116 Hypertonie, akute experimen-telle - 93 
- $\quad$ Augenhintergrund bei - 374Hypertonische Inj ektionenbei

Gehirntumor 374 Hypophysengeschwülste 98

retrobulbärer - 107

die basophilen Adenome 193

I

Idiotie, familiäre amaurotische - und Lipoidstoffwechsel 371

Innere Sekretion und Tapetum 103

Sachregister zu Band 81.

379

Iridenkleisis 183 Iridocyclitis und Spondylarthri-

tis ankylopoetica 359 Iris, Pathologie der--172 (Bericht) Irissarkom, Indikation der Enu-

kleation bei - 89 Iritis auf dentaler Infektion 93

$\mathrm{K}$

Kalziumbehandlung bei Kera-toconjunctivitis phlyctaenulosa

74 Kammerwinkel, Entwicklungs-

geschichte der Gebilde des- 192 Kampimetrie, Lichtpunktwerfer

für - 299 Karzinom des Bulbus 90, 106

- $\quad$ des Augenlids nach Bindehaut-tuberkulose 366

- der Hornhaut 83

Katarakt Entstehung der -

$255 \mathrm{ff}$.

bei Prurigo 362

nichtoperative Behandlung der

- $\quad$ 95Kataraktoperationen 259, 260

- $\quad$ intrakapsuläre 359

- $\quad$ Wert der Knappschen - 368Kayser-Fleischerscher Ring

bei der Pseudosklerose 70 Keratitis filiformis durch Hy-perfunktion der Tränendrüse 272

Keratitis parenchymatosa 72 Keratoconj unctivitis phlyctaenulosa 74

- $\quad$ sicca 272Keratokonus 71Keratoplastik 84Knochenbildung, umschriebene

- $\quad$ in der normalen Sklera 362Knötchenbildung am Pupillar-

saum 175

- $\quad$ der Bindehaut und Hornhautund ihre Beziehung zu denRheumatismusknötchen 19

Kohlehydratuntersuchungen

zur Frage der Blut-Kammer-

wasserschranke 190 Kolloidlehre, medizinische -

196, 275 (Bb.) Konvergenzbewegungen 346 Kupfersplitterverletzungen, intraokulare 100 Kuraufenthalt im Kindesalter

"5

$\mathrm{L}$

Lenticonus posterior 256 Lepra, Keratitis bei - 79 Leukosarkomatose Paltauf-

Sternberg, Augenveränderun-

gen bei der - 308 Lichtpunktwerfer für Perime-

trie und Kampimetrie 299

Lider, Maßnahmen an den - zum Schutze der Hornhaut bei Glotz-augen und Fazialislähmung 13

- $\quad$ Krebs des - nach Bindehaut-tuberkulose 366 
Lidgeschwülste, Behandlung der - 100

Linse, Pathologie der - 254 (Bericht)

- $\quad$ Doppelbrechung der - 94Linsenkapsel, Pinzette zur Ex-

traktion der - 367 Lipoidgranulomatose 372 Lipoidstoffwechsel, Störungen

des - - bei Erbkrankheiten des

Nervensystems 371 Lochbildung, senile der Netz-

und Aderhaut 187 Lokalisationsschablonen aus

Cellon 92 Lumbalanästhesie, Augenmus-

kellähmungen nach - 353 Lympliadenosis aleucaemica der

Conjunctiva 368

- der Orbita 265Lymphogranuloma malignum

der Semilunarfalten 63

$\mathrm{M}$

Medientrübung, Schattenbildung durch -188

Membrana epipapillaris 251

Membrana papillaris persist ens corneae adhaerens 336

Meropitan no

Mikrophthalmus mit Orbital-Oberlidzyste 55

Mikroskopische Technik 276 (Bb.)

Musculi recti externi, ange-borene Lähmung beider - 367

Myasthenie 350

Myopie, die degenerativen Stig-men bei hochgradiger - 109

- $\quad$ und Glaukom 179

N Nasennebenhöhlen und Augen-

erkrankungen 267 (Bericht) Nervus opticus und Carotis in-

terna 92

gesonderte pupillomotorische Fasern im - 35

umschriebene Grubenbildung des Sehnervenkopfes 314

doppelseitige Geschwulst der - 361

Befunde am - bei Stirnhirn-erkrankungen 192

Netzhaut s. Retina Netzhautablösung s. Ablatio

retinae. Neurologische Beziehungen

der Augenkrankheiten 363

380

Sachregister

zu Band 81.

Nystagmus, Beseitigungdes-186

0

Ophthalmia sympathica, Pa-

thogenese der - 107 Ophthalmoskopie im fokalen

und indirekten Licht 274 Optikomyelitis Henneberg3Ô5 Or bit a, Pathologie der - 262

(Bericht) Orbitalgumma 112 Orbital-Oberlidzyste mit An-

ophthalmus 55 Orbitaltumoren 265, 266 Orbitalverletzung 360 Ostitis fibrosa 269

Ostseeheilfaktoren, Einfluß der

- $\quad$ auf allergische Hautreaktio-nen 115 
P Papillom der Cornea, in Karzinom

des Bulbus entartet 90 Parkinsonismus, chronischer

postenzephalitischer - 349 Periarteriitis nodosa tuber-

culosa der Netzhaut 194 Perimetrie, Lichtpunktwerfer für

- 299Phakomatosen, Augensymptome

bei - 372 Phlebolithen bei intermittieren-

dem Exophthalmus 91 Pigmentzyste in der Vorderkam-

mer 91 Pilokarpin 68 Pinzette zur Extraktion der Lin-

senkapsel 367 Prurigo, Katarakt bei - 362 Pseudogliom vorgetäuscht durch

ein Chorioideakolobom mit Glia-

wucherungen 155 Pseudosklerose, Kayser-Flei-

scherscher Ring bei der - 70 Pupillomotorische Fasern, ge-

sonderte - im Sehnerven 35 Puppenaugenbewegungen 346

$\mathrm{R}$

Reflexionslinien, bogenförmige

- von der Papille zur Macula

lutea 363 Regio intergeniculata 105 Retina, atypische Gefäßtuberku-

lose der - 194 - Zyste der - 363 Retinitis circinata, familiäre

Beziehungen bei -164 Retinitis pigmentosa, spontane

Besserung der Sehschärfe bei -

362 Rheumatismusknötchen, Be-

ziehung der - zur Knötchen-

bildung auf Bindehaut und

Hornhaut 19

$\mathrm{S}$

Salvarsan, Blutungen nach - 90

Schädel, Veränderungen am -

und Augenerkrankungen 268

(Bericht)

Schattenbildung durch Medien-

trübung 188 Schielen 347, 348 Schüller-Christiansche

Krankheit 269 Sehnerv s. Nervus opticus Semilunarfalten, Lymphogra-nuloma malignum der 63 Sinusthrombosen 265 Skier a, Pathologie der -87 (Bericht)

blaue 367

umschriebene Knochenbildung in der normalen - 362

senile Entartung der $\mathbf{m}-\cdot$ an den Ansatzstellen der geraden Au-genmuskeln 187

Oberflächengröße der - beim Embryo und Neugeborenen und die Beziehungen zur Megalo-

cornea der Erwachsenen 213

Variabilität der Durchmesser und der Oberflächengröße der - bei Megalo-, Normal- und Mikro-

cornea 132

Skleraltrepanation, Technik

der - 117, 182 Spondylarthritis ankylopoe-

tica und Iridocyclitis 359 Stellungsanomalien 347 Status dystrophicus, Horner-

syndrom und Heterochromie 113 Stillsche Krankheit, Augen-

komplikationen bei - 91 Stirnhirnerkrankungen, Be-

funde am Sehnerven bei - 192 Syphilis und Iritis 174 Syphilis maligna praecox mit 
Keratitis parenchymatosa 73

$\mathrm{T}$

Tapetum undinnere Sekretion 103 Tarsoplastik oder kombinierte

Exzision 108 Tätowage 85

- $\quad$ der Hornhaut mit Goldchlorid356

Therapeutische Unverträg-

lichkeiten 115 Thorium-X-Behandlung des

Xanthelasma 364 Tonsillen als Eintrittspforten für

Tuber kelbazillen 114 Toti-Operation 232 Trachom 79

-- Klinische Frühdiagnose des - 1 Tränenabflußwege, Pathologie

der -101

Namenregister zu Band 81.

381

Tränendrüse, Keratitis filiformis

durch Hyperfunktion der - 272 Tränenpunkt und Tränenröhr-

chen, Untersuchung der - mit

der Spaltlampe 108 Tränensack, Frühdiagnostik ma-

ligner Umbildungen in der Ge-

gend der -- 168 Tuberkelbazillen, Tonsillen als

Eintrittspforten für - 114 Tuberkulom der Episklera 88 Tuberkulose, Häuíigkeit undFor-

men der Augenerkrankungen bei

aktiver - - anderer Organe 197

- $\quad$ Keratitis parenchymatosa bei -

73 Tuberkulose des Auges 174

der Cornea 108

der Bindehaut mit Lidkarzinom 366

Tumor en, Frühdiagnostik malig-ner - in der Tränensackgegend 168

$\ddot{\mathrm{u}}$

Ulcus rodens 77

Ulcus rodens, operativeBehand-

lung des -365 Ulcus serpens $75 \mathrm{ft}$.

V.

Verätzungen der Hornhaut 83 Vorderkammer, Cholesterinab-

lagerung in der - $112 \mathrm{~W}$ Webersches Syndrom 353 Wechselbrille zum Gebrauch für

Feme und Nähe 364 Wimpernhaar in der unteren

Übergangsfalte 363 X Xanthelasma, Thorium-X-Be-

handlung des - $364 \mathrm{Z}$ Zeuge, Arzt als - in der Sozial-

versicherung und Reichsversor-

gung 276 (Bb.) Ziliarkörper, Pathologie des -

172 (Bericht) Zyklodialyse 183 - Technik der - 117

Namenregister zu Band 81.

Abdulaev 266 Abraham 350 Adamantiades 258 Adamson 346 Adrian-Matschke92 Adrogué Ï72, 256 Alajamo 80 Andina 84 Apetz 175 Archangelskij 263 Arnold 356 Asaoka 265 Attie 259 Aust 188 d'Autrevaux 73 Avizonis 1 Azerad 264

B 
Bader 375 Badot 257 Bailliard 180 Balado 266 Baquis 263, 266 Barberi 255 Bardanzellu 268 Barkan 260 Barrada 266 Barraquer 348

Barrenchea 259 Barrow 175 Bartels 84, 346, 357 Bartók 107 Bassin 263 Bauer 269 Beauchesne 256 Beigelmann 82 Beisbarth 347 Belot 258 Bender 55, 178 Benedict 176 Berliner 83 Bertoldi 174, 175 Beyne 347 Biantroskaja 175 Bielschowsky 351 Biggom 353 Biró 362 v. Blaskovics 13, 365 ,

366, 368, 370 Böck 190 Bonnet 267 Boquien 269 Bormacher 265 Bottrup 85 Boulin 264 Brana 109 Brandau 374 Brobeck 264

Brugger 319 Brüning 115 Bruzzone 269 Bucklers 81, 176 Buhss 104 Bunge 183 Burch 266

Burnier 455 Busacca 175 Butler 184, 352, 353

C. C Campbell 264 Canneyt 174 Carle 260 Cattaneo 262 Ceelen 372 Chaptal 374 Charlin 179 , 269 Chester 269 Chotzen 268 Claes 185 Clausen 95, 347 Cocuzza 181 Cohen 350 Colomba 178 Coppez 80, 185, 351 Cottenot 259 Cowan 263 Cozzoli 255 